\title{
Comparison of tadalafil pharmacokinetics after administration of a new orodispersible film versus a film-coated tablet
}

This article was published in the following Dove Press journal: Drug Design, Development and Therapy

\author{
Sang-In Park, ${ }^{1, *}$ Su-Hak \\ Heo, ${ }^{2,3, *}$ Gihwan Kim, ${ }^{2}$ \\ Seokhoon Chang, ${ }^{2}$ Keon- \\ Hyoung Song, ${ }^{3}$ Min-Gul Kim, ${ }^{4}$ \\ Eun-Heui Jin, ${ }^{5}$ JaeWoo Kim, ${ }^{5}$ \\ SeungHwan Lee,' Jang Hee \\ Hong ${ }^{5,6}$ \\ 'Department of Clinical Pharmacology and \\ Therapeutics, Seoul National University \\ College of Medicine and Hospital, Seoul, \\ Republic of Korea; ${ }^{2}$ R\&D Center, C.L. \\ Pharm Co., Ltd, Seoul, Republic of Korea; \\ ${ }^{3}$ Department of Pharmaceutical Engineering, \\ College of Medical Science, Soonchunhyang \\ University, Asan, Republic of Korea; \\ ${ }^{4}$ Department of Pharmacology, School of \\ Medicine, Chonbuk National University, \\ Jeonju, Republic of Korea; ${ }^{5}$ Clinical Trials \\ Center, Chungnam National University \\ Hospital, Daejeon, Republic of Korea; \\ ${ }^{6}$ Department of Pharmacology, College of \\ Medicine, Chungnam National University, \\ Daejeon, Republic of Korea \\ *These authors contributed equally \\ to this work
}

Correspondence: SeungHwan Lee Department of Clinical Pharmacology and Therapeutics, Seoul National University College of Medicine and Hospital, I0I Daehak-ro, Jongno-gu, Seoul 03080, Republic of Korea Tel +82 220722343

Fax +8227429252

Email leejh413@snu.ac.kr

Jang Hee Hong

Clinical Trials Center (5F, Biomedical

Convergence Research Center), Chungnam National University Hospital, 266 Munhwa-ro, Jung-gu, Daejeon, 350I5, Republic of Korea

Tel +82 422806940

Fax +82 422806947

Email boniii@cnu.ac.kr
Background: An orodispersible film (ODF) of tadalafil may provide increased convenience for erectile dysfunction (ED) patients as compared to conventional tablet formulations. In this study, we aimed to compare the pharmacokinetic, safety, and tolerability profiles of a newly developed ODF formulation of tadalafil to those of a film-coated tablet (FCT) of tadalafil.

Materials and methods: This study was conducted in healthy male subjects using an openlabel, randomized sequence, two-period, two-formulation, single-dose, crossover design. The subjects were randomly assigned to one of two sequences of the two formulations: both the test drug (ODF) and the reference drug (FCT) contained $20 \mathrm{mg}$ of tadalafil. Blood samples were collected up to $72 \mathrm{~h}$ after administration. Plasma concentrations of tadalafil were analyzed using liquid chromatography-tandem mass spectrometry. Geometric mean ratios (GMRs) of the ODF to FCT formulations and their $90 \%$ CIs for the pharmacokinetic parameters were estimated. Safety and tolerability were assessed throughout the study.

Results: Forty healthy male subjects were enrolled, and 36 of these completed the study. The GMRs (90\% CIs) of the maximum plasma concentration and the area under the plasma concentration-time curve from time zero to the time of the last quantifiable concentration for tadalafil were 0.927 (0.882-0.974) and 0.972 (0.918-1.029), respectively. Both ODF and FCT formulations were well tolerated, and no clinically significant changes from the baseline were observed after dosing.

Conclusion: The pharmacokinetics of the tadalafil ODF formulation did not differ significantly from those of the FCT formulation. Furthermore, the safety and tolerability profiles of the ODF formulation were comparable to those of the FCT formulation. Therefore, this tadalafil ODF formulation offers a convenient treatment option for patients with erectile dysfunction.

Keywords: erectile dysfunction, orodispersible film, tadalafil, pharmacokinetics

\section{Introduction}

Erectile dysfunction (ED) is the inability to attain and maintain erection as part of the overall multifaceted process of male sexual function. ${ }^{1}$ ED is an important worldwide health issue that affects nearly $20 \%-40 \%$ of men between the ages of 60 and 69 years, and more than $50 \%$ of men older than 70 years. ${ }^{2-5}$ A previous study reported that ED is significantly and independently associated with an increased risk of coronary heart disease, stroke, other cardiovascular diseases, and all-cause mortality. ${ }^{6}$ In addition, ED compromises quality of life and is associated with depression, anxiety, and a loss of self-esteem, and thus requires effective treatment. ${ }^{1}$ Tadalafil (Cialis ${ }^{\circledR}$; Eli Lilly and Company, Indianapolis, IN, USA) is an oral therapy for ED that acts as a selective inhibitor of cyclic guanosine monophosphate-specific phosphodiesterase 
type 5 (PDE5). ${ }^{7}$ Since its launch in 2003, tadalafil has been formulated as yellow film-coated tablets (FCTs) for oral administration that contain $2.5 \mathrm{mg}, 5 \mathrm{mg}, 10 \mathrm{mg}$, or $20 \mathrm{mg}$ tadalafil ${ }^{7}$ this formulation can be administered with water intake, which can be inconvenient for ED patients.

An orodispersible film (ODF), a relatively new dosage formulation, is a postage stamp-sized strip of thin polymeric film that disintegrates or dissolves almost instantaneously when placed onto the tongue. ${ }^{8,9}$ An ODF can dissolve and disintegrate in the mouth in less than $1 \mathrm{~min}$, without water intake or swallowing. ${ }^{8,10}$ In addition, administration using an ODF has been shown to improve the clinical performance of drugs, as compared to existing formulations such as tablets or capsules. ${ }^{8,11}$ The taste and performance of an ODF can be improved by altering the ingredients, including the film-forming polymers, active pharmaceutical compounds, plasticizers, flavors, and sweeteners. ${ }^{12}$ For example, masking the original taste of active pharmaceutical ingredients is important because the formulation needs to be acceptable and convenient. Moreover, an ODF can improve compliance, as compared to conventional oral formulations, by eliminating the risk of choking, offering accurate dosing, and allowing administration without water. ${ }^{8}$ Considering that the prevalence of ED increases with aging, an ODF can especially enhance the compliance of geriatric patients with swallowing difficulties. Furthermore, owing to recent developments in ODF manufacturing technology, an ODF can now provide a cost-effective alternative to conventional dosage forms. ${ }^{8,13}$

Orodispersible formulations of other PDE5 inhibitors that are approved for ED treatment, including sildenafil and vardenafil, have been developed for enhanced dosing convenience. ${ }^{14,15}$ A previous study suggested that a new ODF of sildenafil can be used interchangeably with the conventional FCT based on the bioequivalence between the two formulations. ${ }^{14}$ In addition, a new orodispersible tablet of vardenafil showed a rapid onset of action that was comparable to that of vardenafil FCT. ${ }^{15}$ Tadalafil has a different chemical structure from sildenafil and vardenafil, which is reflected in its pharmacokinetic profile. ${ }^{16}$ For instance, tadalafil has a longer half-life than sildenafil or vardenafil, leading to a prolonged period of efficacy.,17,18 Additionally, the rate and extent of absorption of tadalafil are unaffected by food intake. ${ }^{16,19}$ Therefore, an ODF formulation of tadalafil may provide more therapeutic options to address the needs and preferences of ED patients. Based on these requirements, a new ODF containing $20 \mathrm{mg}$ tadalafil was developed (C.L. Pharm Co., Ltd, Seoul, Republic of Korea) to provide increased convenience for ED patients, as compared to conventional tablet formulations. In order to provide a viable alternative, the pharmacokinetic profile of tadalafil ODF, as well as its safety and tolerability profiles, should be comparable to those of a conventional tadalafil tablet formulation.

In this context, the present study was conducted in healthy subjects to compare the pharmacokinetic, safety, and tolerability profiles of the newly developed tadalafil ODF formulation, taken without water, to those of a conventional formulation of tadalafil, taken with water. This study was registered with the identifier number KCT0002360 at the Clinical Research Information Service (CRIS, http://cris. nih.go.kr).

\section{Materials and methods}

The study was approved by the Ministry of Food and Drug Safety and the Institutional Review Board of Chungnam National University Hospital (Daejeon, Republic of Korea), and was conducted in accordance with the Declaration of Helsinki for biomedical research involving human subjects and with the Guideline for Good Clinical Practice. A detailed explanation of the study was provided to each participant, and their written informed consent was obtained prior to screening.

\section{Subjects}

The sample size was calculated based on the within-subject coefficient of variation for the pharmacokinetic parameters (maximum plasma concentration $\left[\mathrm{C}_{\max }\right]$ and area under the plasma concentration-time curve [AUC]) of tadalafil reported in a previous study. ${ }^{20}$ Taking the dropout rate into consideration, it was estimated that a total of 40 subjects would be appropriate to detect a $20 \%$ difference between the test and reference formulations at a 5\% significance level, with $90 \%$ power.

Healthy male volunteers without ED aged 19-45 years who weighed $\geq 55 \mathrm{~kg}$ and were within $20 \%$ of the ideal body weight for their height were eligible for the study. The health of each subject was confirmed by physical examination, measurement of vital signs, 12-lead electrocardiography, and routine laboratory assessments (hematology, chemistry, serology, and urinalysis); these were performed within 4 weeks before the first study drug administration.

All subjects with a tadalafil-associated allergy history were excluded from this study. Subjects with evidence of, or a history of, clinically significant hepatic, pancreatic, renal, neurological, pulmonary, endocrine, hematological, psychiatric, cardiovascular, ocular, or genitourinary diseases were excluded. Subjects with any underlying condition or 
disease that would interfere with absorption of the study drug were excluded. Additionally, subjects were also excluded if they had received any prescription or herbal medications within 2 weeks and any over-the-counter medications within 7 days before the study. Subjects with a history of regular alcohol consumption exceeding 21 units/week ( 1 unit $=10 \mathrm{~g}$ of pure alcohol) or drug abuse were excluded. Subjects were excluded if they participated in another clinical study within the 2 months preceding the first dose of study medication. Subjects were excluded if they had donated blood (excluding plasma donations) within the 2 months prior to dosing, or had donated plasma within 1 month prior to dosing. Negative serology test results for hepatitis B surface antigen and antibody, anti-hepatitis $\mathrm{C}$ virus antibody, and HIV antigen-antibody were required for inclusion in this study. Subjects were excluded if they had any surgical or medical conditions that could significantly alter the pharmacokinetics of the study drugs. Subjects were asked to avoid smoking and consuming caffeinated foods or beverages during the hospitalization periods. Subject compliance with these restrictions was identified by self-reporting and by taking the subject's history.

\section{Study design}

This study was conducted using an open-label, randomized sequence, two-period, two-formulation, single-dose, singlecenter, crossover design. All of the subjects were randomly assigned to one of two sequences of the two formulations: $20 \mathrm{mg}$ tadalafil ODF (lot no TAD14001, expiry 2016.09.15; C.L. Pharm Co., Ltd) as the test drug and $20 \mathrm{mg}$ tadalafil FCT (lot no C289838, expiry 2016.11.13; Eli Lilly and Company) as the reference drug. Each of the sequence groups (A and B) consisted of 20 subjects. The subjects were admitted on the day prior to dosing (Day -1) and fasted for $10 \mathrm{~h}$ prior to receiving the drugs. The baseline evaluations were performed prior to dosing. During the first period, subjects from the A sequence group received $20 \mathrm{mg}$ tadalafil FCT with $240 \mathrm{~mL}$ water, whereas subjects from the B sequence group received $20 \mathrm{mg}$ tadalafil ODF without water after swallowing $20 \mathrm{~mL}$ of water in order to wet the mouth. ${ }^{21}$ Subjects were discharged from the study center on Day 2, and pharmacokinetic samples were taken at $48 \mathrm{~h}$ and $72 \mathrm{~h}$ in an outpatient clinic. After a 1-week washout period, the A sequence group received $20 \mathrm{mg}$ tadalafil ODF and the B sequence group received $20 \mathrm{mg}$ tadalafil FCT, as described above.

\section{Safety and tolerability assessments}

Adverse events (AEs) were recorded, preferably with diagnosed medical terms rather than symptoms or laboratory values; if this was not possible, terms for symptoms and signs that investigators observed or subjects reported were used. AEs were generally coded using the preferred terms employed by the Medical Dictionary for Regulatory Activities (Version 18.1). Physical examinations, 12-lead electrocardiography, routine laboratory assessments (hematology, chemistry, and urinalysis), and vital sign measurements were performed at predefined regular intervals throughout the study.

\section{Pharmacokinetic assessments}

Blood samples were taken from an indwelling cannula inserted into a forearm vein at the following scheduled times: 0 (before administration), 0.33, 0.67, 1, 1.5, 2, 2.5, $3,4,6,8,12,24,48$, and $72 \mathrm{~h}$ after dosing with both the test and the reference drugs. Eight milliliters of blood were drawn into EDTA-coated tubes after the first $1 \mathrm{~mL}$ had been discarded from the intravenous cannula, to avoid normal saline contamination. Samples were centrifuged at $1,811 \times g$ $(3,000 \mathrm{rpm})$ for $10 \mathrm{~min}$ at $4^{\circ} \mathrm{C}$, and the supernatant (plasma) was immediately stored in polypropylene tubes at $-70^{\circ} \mathrm{C}$ until analysis.

\section{Bioanalytical methods}

Plasma concentrations of tadalafil were determined using validated liquid chromatography (Thermo Dionex UltiMate 3000; Thermo Fisher Scientific, Waltham, MA, USA) coupled with tandem mass spectrometry (Thermo TSQ Vantage; Thermo Fisher Scientific). A $50-\mu \mathrm{L}$ aliquot of plasma and $50 \mu \mathrm{L}$ of internal standard solution $(100 \mu \mathrm{g} / \mathrm{L}$ Tadalafil-d 3 in $50 \%$ acetonitrile; Toronto Research Chemicals Inc., Toronto, ON, Canada) were added to $400 \mu \mathrm{L}$ acetonitrile. The mixture was vortexed for $5 \mathrm{sec}$ and then centrifuged approximately at $14,500 \mathrm{~g}$ for $5 \mathrm{~min}$ at $4^{\circ} \mathrm{C}$. A $5-\mu \mathrm{L}$ aliquot of the diluted supernatant was injected onto the analytical column for liquid chromatography-tandem mass spectrometry analysis. During prestudy validation, calibration curves for both analyses in human plasma were obtained using calibration standards ranging from 2 to $1,000 \mu \mathrm{g} / \mathrm{L}$ tadalafil, each of which was freshly prepared in-house and protein-precipitated together with blank plasma samples and quality control samples in each analytic run. Quality control samples for tadalafil were used to assess the intraday and interday precision, accuracy, recovery, and stability of the analysis. These samples were prepared in-house on the day that the first study samples were received, and stored below $-70^{\circ} \mathrm{C}$ with the study samples.

Chromatographic separation was performed using a Hypersil GOLD column $(100 \times 2.1 \mathrm{~mm}, 1.9 \mu \mathrm{m}$; Thermo Fisher Scientific). The column was eluted with a mobile phase consisting of $0.1 \%$ ammonium formate and acetonitrile 
$(20: 80, \mathrm{v} / \mathrm{v})$ at a flow rate of $0.3 \mathrm{~mL} / \mathrm{min}$. Multiple reaction monitoring analysis was applied to detect ion transitions at $\mathrm{m} / \mathrm{z} 390.4 \rightarrow 268.2$ for tadalafil and $\mathrm{m} / \mathrm{z} 393.4 \rightarrow 271.2$ for the internal standard. The lower limit of quantification was $2 \mu \mathrm{g} / \mathrm{L}$ and the calibration was linear in the range from 2 to $1,000 \mu \mathrm{g} / \mathrm{L}\left(r^{2} \geq 0.9953\right)$. Within-run precision and accuracy were determined by repeated analysis of tadalafil over the concentration range of lower limit of quantification $(2 \mu \mathrm{g} / \mathrm{L})$, low $(6 \mu \mathrm{g} / \mathrm{L})$, medium $(60 \mu \mathrm{g} / \mathrm{L})$, and high $(800 \mu \mathrm{g} / \mathrm{L})$ quality control samples. A total of five replicates of each quality control concentration were analyzed. Between-run precision and accuracy were determined from a total of three replicates of each quality control concentration. The intrabatch and interbatch coefficients of variation of tadalafil were $\leq 4.36 \%$ and $\leq 6.96 \%$, respectively. The intrabatch and interbatch accuracies ranged from 94.45 to $97.36 \%$ and from 98.12 to $100.88 \%$, respectively.

\section{Pharmacokinetic analyses}

The individual pharmacokinetic parameters were obtained by noncompartmental methods using Phoenix WinNonlin ${ }^{\circledR}$ (Version 6.4; Pharsight Corporation, St Louis, MO, USA). The $\mathrm{C}_{\max }$ and time to $\mathrm{C}_{\max }\left(\mathrm{T}_{\max }\right)$ were obtained directly from the observed values. The AUC from time zero to the time of the last quantifiable concentration $\left(\mathrm{AUC}_{\text {last }}\right)$ was calculated using the linear up/log down trapezoidal rule. The elimination rate constant $\left(\lambda_{\mathrm{z}}\right)$ describes the rate of decrease in concentration per unit time; this was estimated from the log-linear terminal part of the concentration-time curve as the slope of the natural logarithm of concentration against time. The AUC from time zero to infinity $\left(\mathrm{AUC}_{\text {inf }}\right)$ was calculated using the following equation: $\mathrm{AUC}_{\text {last }}+\mathrm{C}_{\text {last }} / \lambda$, where $\mathrm{C}_{\text {last }}$ was the last measurable concentration. The elimination half-life $\left(t_{1 / 2}\right)$ was calculated as the natural logarithm of 2 divided by $\lambda_{z}$. The apparent clearance $(\mathrm{CL} / \mathrm{F})$ was calculated as the dose divided by the $\mathrm{AUC}_{\text {inf }}$.

\section{Statistical analysis}

The statistical analysis was performed by $\operatorname{SAS}^{\circledR}$ (Version 9.3; SAS Institute Inc., Cary, NC, USA). Descriptive statistics, including the mean \pm standard deviation, were used to summarize the pharmacokinetic data for the two formulations. To compare the pharmacokinetics of ODF and FCT, the geometric mean ratio (GMR) (ODF/FCT) and the $90 \%$ CIs of $\mathrm{C}_{\max }, \mathrm{AUC}_{\text {last }}$, and $\mathrm{AUC}_{\text {inf }}$ were assessed by analysis of variance using a mixed effect model. Formulation, sequence, and period were used as fixed effects, and a subject nested within the sequence was used as a random effect. If the
$90 \%$ CIs of these GMRs were entirely within the conventional bioequivalence range of $0.8-1.25$, the bioavailability of the two tadalafil formulations was considered to be comparable. $p<0.05$ was considered statistically significant.

\section{Results \\ Demographic characteristics}

In total, 40 healthy males were enrolled and randomized into experimental groups. The ages, weights, and heights (mean \pm standard deviation) of the enrolled subjects were $23.1 \pm 2.1$ years, $68.9 \pm 7.7 \mathrm{~kg}$, and $173.7 \pm 5.4 \mathrm{~cm}$, respectively (Table 1). The demographic characteristics were not significantly different between the sequence groups, with $p$-values of $0.667,0.661$, and 0.893 for age, weight, and height, respectively (Wilcoxon rank-sum test or independent $t$-test). Four subjects were withdrawn from the study due to protocol violation $(n=1)$; abnormality in creatine phosphokinase $(n=1)$; concomitant drug administration $(n=1)$; or no-show $(n=1)$. Therefore, a total of 36 subjects completed the study as planned. The data relating to the withdrawn subjects were excluded from the pharmacokinetic analysis.

\section{Pharmacokinetics}

The mean plasma tadalafil concentration versus time profiles after administration of tadalafil ODF and FCT are shown in Figure 1. These were superimposable for the two formulations. In addition, the median $\mathrm{T}_{\max }$ and the mean values of $\mathrm{C}_{\text {max }}, \mathrm{AUC}_{\text {last }}, \mathrm{AUC}_{\text {inf }}, \mathrm{t}_{1 / 2}$, and $\mathrm{CL} / \mathrm{F}$ of tadalafil were also comparable between the two formulations, as presented in Table 2. Furthermore, the ODF to FCT GMRs (90\% CIs) for $\mathrm{C}_{\max }$ and $\mathrm{AUC}_{\text {last }}$ were 0.927 (0.882-0.974) and 0.972 (0.918-1.029), respectively; these fell entirely within the conventional bioequivalence range of 0.8-1.25 (Table 2). In addition, no trend was found in our individual comparison of $\mathrm{AUC}_{\text {last }}$ and $\mathrm{C}_{\max }$ (Figure 2).

\section{Safety and tolerability}

There were no serious AEs reported after drug administration. A total of 34 AEs were reported in 27 subjects. Twenty-one

Table I Demographic characteristics of the study subjects

\begin{tabular}{lllll}
\hline Characteristics & Sequence $\mathbf{A}^{\mathbf{a}}$ & Sequence $\mathbf{B}^{\mathbf{b}}$ & $\mathbf{p}$-value & Total \\
\hline Number of subjects & 20 & 20 & & 40 \\
Age (years) & $23.2 \pm 2.3$ & $23.1 \pm 1.8$ & $0.667^{\mathrm{c}}$ & $23.1 \pm 2.1$ \\
Weight $(\mathrm{kg})$ & $69.4 \pm 6.9$ & $68.3 \pm 8.5$ & $0.66 \mathrm{I}^{\mathrm{d}}$ & $68.9 \pm 7.7$ \\
Height $(\mathrm{cm})$ & $173.8 \pm 5.2$ & $173.6 \pm 5.8$ & $0.893^{\mathrm{d}}$ & $173.7 \pm 5.4$ \\
\hline
\end{tabular}

Notes: Values are presented as the mean \pm standard deviation. ${ }^{\text {SSequence }} \mathrm{A}$ : film-coated tablet formulation followed by orodispersible film. 'bequence B: orodispersible film followed by film-coated tablet formulation. 'Wilcoxon rank-sum test. Independent $t$-test. 

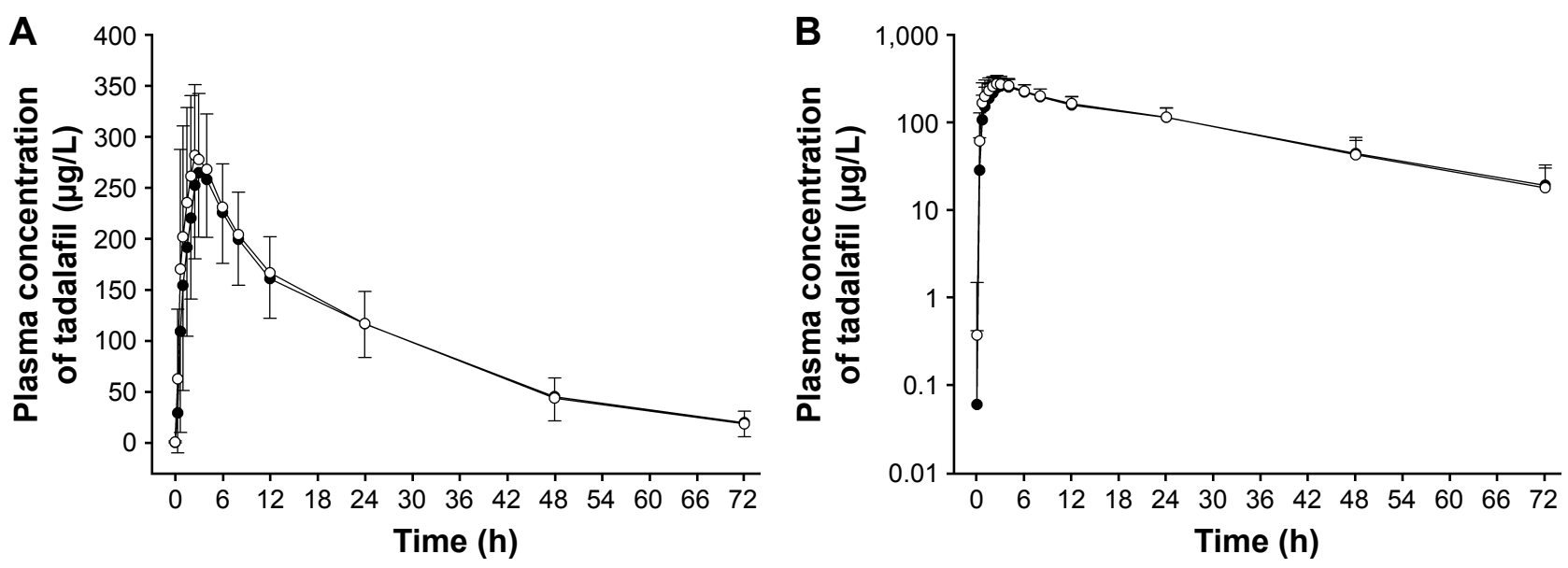

Test drug $-0-$ Reference drug

Figure I Mean tadalafil plasma concentrations versus time profiles in healthy subjects $(n=36)$ after administration of a single dose presented using (A) a linear scale and $(\mathbf{B})$ a semilog scale. Error bars denote the standard deviations. The test drug was an orodispersible film and the reference drug was a film-coated tablet; both contained 20 mg tadalafil.

events occurred in the FCT treatment group and 13 in the ODF treatment group. Twenty of the AEs reported in 18 subjects were considered to be adverse drug reactions, as follows (n values indicate the number of adverse drug reactions): headaches in the ODF $(n=4)$ and FCT $(n=4)$ groups; hot flush in the ODF ( $\mathrm{n}=3)$ and FCT $(\mathrm{n}=5)$ groups; back pain in the ODF $(n=1)$ and FCT $(n=1)$ groups; chest discomfort in the FCT group ( $\mathrm{n}=1)$; and palpitations in the FCT group $(\mathrm{n}=1)$. All of these adverse drug reactions were considered to be mild in intensity and resolved spontaneously. No statistically significant differences in the numbers of subjects who experienced AEs were found between the two treatment groups ( $p=0.232$, Fisher's exact test). No clinically significant findings were observed upon physical examination, including changes in vital signs, electrocardiography, or clinical laboratory evaluations.

\section{Discussion}

In this study, we evaluated the pharmacokinetics of $20 \mathrm{mg}$ tadalafil when administered to healthy subjects as a new ODF formulation or as a FCT. This study indicated that $\mathrm{C}_{\max }$ and $\mathrm{AUC}_{\text {last }}$ were comparable for the ODF and FCT formulations. The GMRs for $\mathrm{C}_{\max }$ and $\mathrm{AUC}_{\text {last }}$ were 0.927 and 0.972 , respectively, and their $90 \%$ CIs were within the conventional bioequivalence range of 0.80-1.25 (Table 2). Furthermore, pharmacokinetic parameters including $\mathrm{T}_{\max }, \mathrm{AUC}_{\mathrm{inf}}, \mathrm{t}_{1 / 2}$, and $\mathrm{CL} / \mathrm{F}$ of tadalafil were also comparable between the two formulations (Table 2). In addition, the mean plasma concentration-time profiles were similar for the two formulations of tadalafil from predose $(0 \mathrm{~h})$ to $72 \mathrm{~h}$ after dosing (Figure 1). Moreover, no specific trend or systematic deviation was found in our comparison of the individual $\mathrm{AUC}_{\text {last }}$ and $\mathrm{C}_{\text {max }}$ values (Figure 2). Based on these results, the bioavailability

Table 2 Pharmacokinetic parameters of tadalafil after a single oral administration of $20 \mathrm{mg}$ tadalafil as an orodispersible film or as a film-coated tablet

\begin{tabular}{llll}
\hline $\begin{array}{l}\text { Pharmacokinetic } \\
\text { parameters }\end{array}$ & $\begin{array}{l}\text { Orodispersible film } \\
(\mathbf{n}=\mathbf{3 6})\end{array}$ & $\begin{array}{l}\text { Film-coated tablet } \\
(\mathbf{n}=\mathbf{3 6})\end{array}$ & $\begin{array}{l}\text { Geometric mean } \\
\text { ratio }^{\mathbf{a}}\end{array}$ \\
\hline $\mathrm{T}_{\text {max }}(\mathrm{h})$ & $2.5[1.0-6.0]$ & $2.5[0.7-4.0]$ & $0.927(0.882-0.974)$ \\
$\mathrm{C}_{\max }(\mu \mathrm{g} / \mathrm{L})$ & $284.0 \pm 58.5(20.6)$ & $308.1 \pm 65.6(21.3)$ & $0.972(0.918-1.029)$ \\
$\mathrm{AUC}_{\text {last }}(\mu \mathrm{g} \cdot \mathrm{h} / \mathrm{L})$ & $6,544.8 \pm 1,861.9(28.4)$ & $6,693.6 \pm 1,675.4(25.0)$ & $0.978(0.920-1.039)$ \\
$\mathrm{AUC}_{\text {inf }}(\mu \mathrm{g} \cdot \mathrm{h} / \mathrm{L})$ & $7,124.7 \pm 2,349.8(33.0)$ & $7,225.0 \pm 2,084.1(28.8)$ & \\
$\mathrm{t}_{\mathrm{I} / 2}(\mathrm{~h})$ & $17.7 \pm 4.8(26.9)$ & $17.4 \pm 4.5(25.6)$ & \\
$\mathrm{CL} / \mathrm{F}(\mathrm{L} / \mathrm{h})$ & $3.1 \pm 0.9(30.3)$ & $3.0 \pm 0.9(31.2)$ & \\
\hline
\end{tabular}

Notes: Values are presented as arithmetic mean \pm standard deviation (coefficient of variation\%) except for $T_{\max }$, for which median [minimum-maximum] values are presented. a Geometric mean ratio of orodispersible film to film-coated tablet formulation $(90 \% \mathrm{Cl})$.

Abbreviations: $\mathrm{T}_{\max }$, time to maximum plasma concentration; $\mathrm{C}_{\max }$, maximum plasma concentration; $A \mathrm{C}_{\text {last }}$, area under the plasma concentration-time curve from time zero to the time of the last quantifiable concentration; $\mathrm{AUC}_{\text {inf }}$ area under the plasma concentration-time curve from time zero to infinity; $\mathrm{t}_{1 / 2}$, terminal half-life; $\mathrm{CL} / \mathrm{F}$, apparent clearance. 

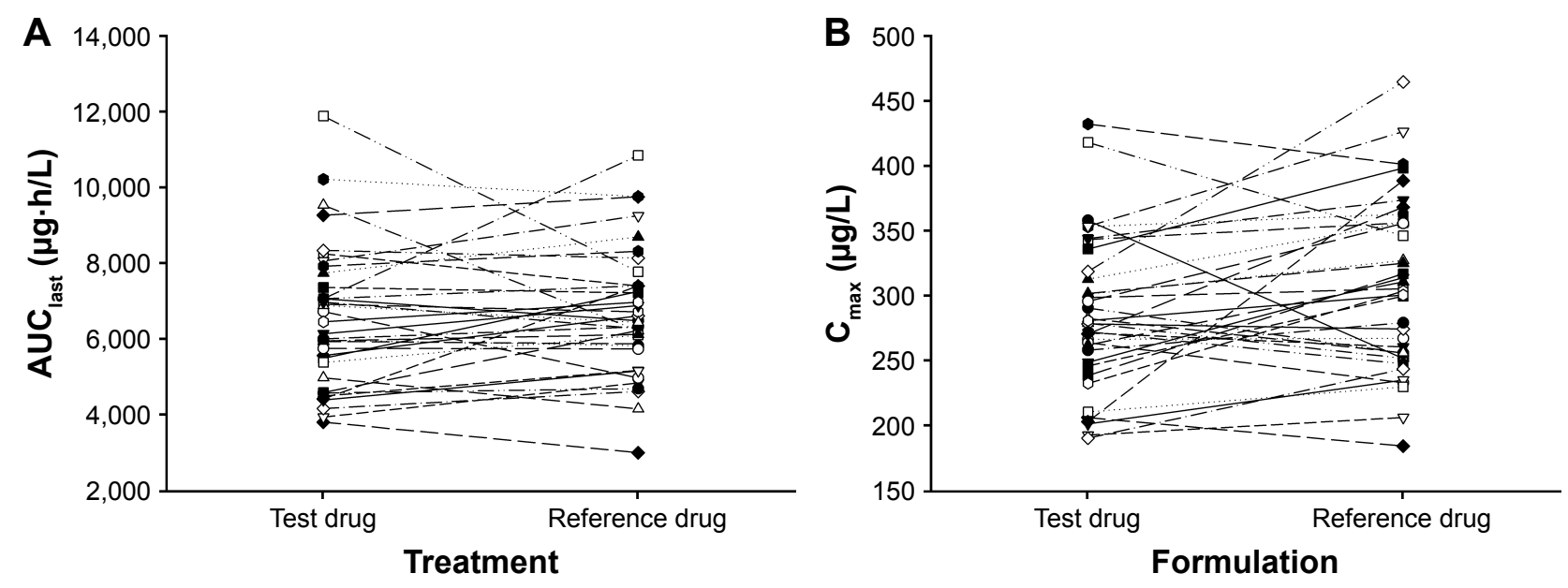

Figure 2 Individual values for $(\mathbf{A})$ the area under the plasma tadalafil concentration-time curve from time zero to the time of the last quantifiable concentration $\left(A \cup C_{\text {last }}\right.$ ) and $(\mathbf{B})$ the maximum plasma tadalafil concentration $\left(\mathrm{C}_{\max }\right)$ after single-dose administration. The test drug was an orodispersible film and the reference drug was a film-coated tablet; both contained $20 \mathrm{mg}$ tadalafil.

of the tadalafil ODF formulation can be concluded to be comparable to that of marketed FCT formulations.

This study was conducted as a two-way crossover design, which is a generally accepted method for bioequivalence studies. With the blood-sampling time points set from predose to $72 \mathrm{~h}$ after dosing, we could fully characterize the tadalafil disposition and absorption phases. Furthermore, the 7-day washout employed between the two study periods was considered sufficient in that it was more than five times the $t_{1 / 2}$ of tadalafil, and no carryover effect was found in the second phase of the study.

The pharmacokinetic properties of tadalafil presented in this study were consistent with those reported previously. A previous study showed that after the administration of a single 20-mg dose, tadalafil was rapidly absorbed with a geometric mean $\mathrm{C}_{\text {max }}$ of $297 \mu \mathrm{g} / \mathrm{L}$ and a median $\mathrm{T}_{\max }$ of $2.0 \mathrm{~h}$; thereafter, the mean concentration-time profiles declined with a geometric mean $\mathrm{t}_{1 / 2}$ of $17.3 \mathrm{~h}$, which was consistent with our study results (Table 2 and Figure 1). ${ }^{20}$

In a previous study, food had negligible effects on the rate and extent of tadalafil absorption. ${ }^{20}$ This indicates that the two formulations of tadalafil used in this study are likely to show similar bioavailabilities, regardless of food intake. Additionally, ED is an age-related chronic condition that is more prevalent in smokers than in nonsmokers. ${ }^{22,23}$ Hence, it is important to consider whether the findings of this healthy volunteer study are applicable to older people and/or smokers. A population pharmacokinetic analysis reported that neither age nor smoking status had significant effects on the systemic exposure of tadalafil. ${ }^{24}$ According to the tadalafil drug label, no dose adjustment is warranted based on age or smoking status. ${ }^{7,20}$ Therefore, the pharmacokinetic profiles of tadalafil observed after administering the ODF or FCT formulations employed in this study are likely to be applicable to elderly ED patients, irrespective of their smoking status, However, considering that the present study was conducted in a limited number of healthy volunteers, further studies involving a larger number of patients would be helpful in order to investigate the effects of factors such as age and/or smoking status on the pharmacokinetic profiles of the two formulations of tadalafil.

In the context of compliance improvement, both safety and convenience are important factors to be considered in clinical use. In relation to safety, our study found that the ODF formulation and the marketed FCT formulation had comparable safety profiles. We found that tadalafil was well tolerated after administration as either the ODF or FCT formulation, and no clinically significant changes from the baseline were observed after dosing. Regarding convenience, the ODF formulation has advantages over conventional tablet formulations because it dissolves rapidly in the oral cavity, without the need to drink water. The ODF formulation could therefore be easier to use, particularly for elderly patients who have difficulties in swallowing tablets. ${ }^{25}$

Taken together, the findings of this study suggest that the ODF and FCT formulations containing $20 \mathrm{mg}$ tadalafil exhibited comparable plasma level-time profiles, and the $90 \%$ CIs of the GMR indicated bioequivalence. Therefore, the present tadalafil ODF formulation has the potential to provide a more convenient alternative to the FCT formulation for ED patients.

\section{Conclusion}

This study was conducted to compare the pharmacokinetics of the newly developed tadalafil ODF, which can be 
administered without water intake, and the conventional FCT formulation. The tadalafil ODF formulation exhibited pharmacokinetic, safety, and tolerability profiles that were comparable to those of the FCT formulation. Therefore, this convenient tadalafil ODF formulation, which can be taken without the need for water or chewing, offers both physicians and patients a novel and attractive option for the treatment of ED.

\section{Acknowledgments}

The authors thank Taewan Kim, Junryang Kim, Hae Jeong, and Kangmo Sung, who are employees of C.L. Pharm Co., Ltd, Seoul, Republic of Korea, for contributions to the preparation of our study. The authors also thank Guangjin Choi, College of Medical Science, Soonchunhyang University, Asan, Republic of Korea, for contributions to the study drug development. This study was sponsored by C.L. Pharm Co., Ltd, Seoul, Republic of Korea. This study was supported by a Korea Health Technology R\&D Project grant from the Korea Health Industry Development Institute, funded by the Ministry of Health \& Welfare, Republic of Korea (grant number HI14C1063). This work was also supported by the research fund of Chungnam National University and by the Basic Science Research Program through the National Research Foundation of Korea, funded by the Ministry of Education (NRF-2017R1D1A1B04033515).

\section{Disclosure}

Su-Hak Heo, Gihwan Kim, and Seokhoon Chang are employees of C.L. Pharm Co., Ltd, Seoul, Republic of Korea. None of the other authors have any conflict of interest to disclose related to this study.

\section{References}

1. NIH Consensus Conference. Impotence. NIH Consensus Development Panel on Impotence. JAMA. 1993;270(1):83-90.

2. Braun M, Wassmer G, Klotz T, Reifenrath B, Mathers M, Engelmann U. Epidemiology of erectile dysfunction: results of the 'Cologne Male Survey'. Int J Impot Res. 2000;12(6):305-311.

3. Nicolosi A, Moreira ED Jr, Shirai M, Bin Mohd Tambi MI, Glasser DB. Epidemiology of erectile dysfunction in four countries: cross-national study of the prevalence and correlates of erectile dysfunction. Urology. 2003;61(1):201-206.

4. Pinnock CB, Stapleton AM, Marshall VR. Erectile dysfunction in the community: a prevalence study. Med J Aust. 1999;171(7):353-357.

5. Shamloul R, Ghanem H. Erectile dysfunction. Lancet. 2013;381(9861): $153-165$.

6. Dong JY, Zhang YH, Qin LQ. Erectile dysfunction and risk of cardiovascular disease: meta-analysis of prospective cohort studies. $\mathrm{J} \mathrm{Am} \mathrm{Coll}$ Cardiol. 2011;58(13):1378-1385.
7. Cialis ${ }^{\circledR}$ (tadalafil) [prescribing information]. Indianapolis, IN: Eli Lilly and Company; 2017. https://www.accessdata.fda.gov/drugsatfda_docs/ label/2017/021368s029lbl.pdf. 2017. Accessed September 20, 2017.

8. Hoffmann EM, Breitenbach A, Breitkreutz J. Advances in orodispersible films for drug delivery. Expert Opin Drug Deliv. 2011;8(3):299-316.

9. Hariharan M, Bogue A. Orally dissolving film strips (ODFS): the final evolution of orally dissolving dosage forms. Drug Deliv Technol. 2009; 9:24-29.

10. Pandya K. Fast dissolving films: a novel approach to oral drug delivery. Int. Jour. of Pharmamedix India. 2013;1(2). Available from: https:// pmindexing.com/index.php/IJPI/article/view/90/0\#.WopzzA2eqC0. Accessed December 11, 2017.

11. Dixit RP, Puthli SP. Oral strip technology: overview and future potential. J Control Release. 2009;139(2):94-107.

12. Amin PM, Gangurde AB, Alai PV. Oral film technology: challenges and future scope for pharmaceutical industry. Int $J$ Pharm Pharm Res. 2015;3(3). http://ijppr.humanjournals.com/wp-content/ uploads/2015/06/13.Ms_.-Prakruti-M.-Amin-Prof.-A.-B.-GangurdeMs.-Pranali-V.-Alai.pdf. Accessed September 20, 2017.

13. Karki S, Kim H, Na S-J, Shin D, Jo K, Lee J. Thin films as an emerging platform for drug delivery. Asian J Pharm Sci. 2016;11(5):559-574.

14. Radicioni M, Castiglioni C, Giori A, Cupone I, Frangione V, Rovati S. Bioequivalence study of a new sildenafil $100 \mathrm{mg}$ orodispersible film compared to the conventional film-coated $100 \mathrm{mg}$ tablet administered to healthy male volunteers. Drug Des Devel Ther. 2017;11:1183-1192.

15. Debruyne FM, Gittelman M, Sperling H, Börner M, Beneke M. Time to onset of action of vardenafil: a retrospective analysis of the pivotal trials for the orodispersible and film-coated tablet formulations. $J$ Sex Med. 2011;8(10):2912-2923.

16. Wright PJ. Comparison of phosphodiesterase type 5 (PDE5) inhibitors. Int J Clin Pract. 2006;60(8):967-975.

17. Viagra ${ }^{\circledR}$ (sildenafil citrate) [prescribing information]. New York: Pfizer Inc.; 2017. https://www.accessdata.fda.gov/drugsatfda_docs label/2017/020895s049lbl.pdf. 2017. Accessed December 11, 2017.

18. Levitra (vardenafil hydrochloride) [prescribing information]. Whippany, NJ: Bayer HealthCare Pharmaceuticals Inc.; 2017. https:// www.accessdata.fda.gov/drugsatfda_docs/label/2017/021400s0201bl. pdf. 2017. Accessed December 11, 2017.

19. Ramakrishna NV, Vishwottam KN, Puran S, et al. Quantitation of tadalafil in human plasma by liquid chromatography-tandem mass spectrometry with electrospray ionization. J Chromatogr B Analyt Technol Biomed Life Sci. 2004;809(2):243-249.

20. Forgue ST, Patterson BE, Bedding AW, et al. Tadalafil pharmacokinetics in healthy subjects. Br J Clin Pharmacol. 2006;61(3):280-288.

21. European Medicines Agency. Guideline on the Investigation of Bioequivalence. London: European Medicines Agency; 2010. http://www.ema. europa.eu/docs/en_GB/document_library/Scientific_guideline/2010/01/ WC500070039.pdf. Accessed September 20, 2017.

22. Feldman HA, Goldstein I, Hatzichristou DG, Krane RJ, McKinlay JB. Impotence and its medical and psychosocial correlates: results of the Massachusetts Male Aging Study. J Urol. 1994;151(1):54-61.

23. Mannino DM, Klevens RM, Flanders WD. Cigarette smoking: an independent risk factor for impotence? Am J Epidemiol. 1994;140(11): 1003-1008.

24. Trocóniz IF, Tillmann C, Staab A, Rapado J, Forgue ST. Tadalafil population pharmacokinetics in patients with erectile dysfunction. Eur J Clin Pharmacol. 2007;63(6):583-590.

25. Irfan M, Rabel S, Bukhtar Q, Qadir MI, Jabeen F, Khan A. Orally disintegrating films: a modern expansion in drug delivery system. Saudi Pharm J. 2016;24(5):537-546. 


\section{Publish your work in this journal}

Drug Design, Development and Therapy is an international, peerreviewed open-access journal that spans the spectrum of drug design and development through to clinical applications. Clinical outcomes, patient safety, and programs for the development and effective, safe, and sustained use of medicines are the features of the journal, which

has also been accepted for indexing on PubMed Central. The manuscript management system is completely online and includes a very quick and fair peer-review system, which is all easy to use. Visit http://www.dovepress.com/testimonials.php to read real quotes from published authors.

Submit your manuscript here: http://www.dovepress.com/drug-design-development-and-therapy-journa 